\title{
Plataforma Di@: Direito à Alimentação - Conhecendo a Dinâmica Familiar na Perspectiva da Segurança Alimentar
}

\author{
Paulo A. Parreira Júnior'1, Carolina Martins dos Santos Chagas², Milena Serenini \\ Bernardes $^{3}$, Paulo Henrique Lima, Heitor A. X. Costa ${ }^{1}$ \\ ${ }^{1}$ Departamento de Ciência da Computação - Universidade Federal de Lavras (UFLA) \\ ${ }^{2}$ Departamento de Nutrição - Universidade Federal de Lavras (UFLA) \\ Caixa Postal 3.037 - 37200-000 - Lavras, MG - Brasil \\ ${ }^{3}$ Departamento de Pediatria - Universidade Federal de São Paulo (UNIFESP) \\ 04023-900- São Paulo, SP - Brasil \\ \{pauloa.junior, heitor, carolinachagas\}@ufla.br, miserenini@gmail.com, \\ ph.lima.tecnico@gmail.com
}

\begin{abstract}
The paper describes the assumptions for creating and using the Di@ Platform, whose objective is to understand the dynamics of families focused on the perception and experience of food insecurity and hunger at home. The Di@Platform emerged from a real demand from Lavras/MG, in the context of Covid-19, and is characterized as a mobile, innovative and unprecedented resource. The results indicate ease in the maintenance, authentication and sending of the interviews by the users and, still, convergence with the proposed goals. The project is expected to serve as a theoreticalmethodological reference for other strategies.

Resumo. $O$ artigo descreve os pressupostos de criação e utilização da plataformaDi@, cujo objetivo é conhecer a dinâmica das famílias com foco na percepção e na vivência de insegurança alimentar e fome no nível domiciliar.A plataformaDi@surgiu de uma demanda real do município de Lavras/MG, no contexto da Covid-19, e se caracteriza como um recurso mobile, inovador e inédito. Os resultados indicam facilidade na manutenção, autenticação e envio das entrevistas pelos usuários e, ainda, convergência com os objetivos propostos. Espera-se que o projeto sirva de referência teórico-metodológica para outras estratégias.
\end{abstract}

\section{Introdução}

Segurança Alimentar e Nutricional (SAN) é compreendida como “[...] a realização do direito de todos ao acesso regular e permanente a alimentos de qualidade, em quantidade suficiente, sem comprometer o acesso a outras necessidades essenciais, tendo como base práticas alimentares promotoras de saúde que respeitem a diversidade cultural e que sejam ambiental, cultural, econômica e socialmente sustentáveis" [Brasil 2006]. 
Considerando a multidimensionalidade da SAN e a complexificação do sistema alimentar $^{1}$, constata-se que muitas são as variáveis de investigação com foco nos resultados, nos processos ou em ambos. Destaca-se aqui a avaliação de processos, pois seu escopo centra-se na investigação do funcionamento de programas, buscando a identidade entre ação e resultado [Panelli-Martins et al. 2008].

Preocupações com a escassez de alimentos ou a experiência com a situação de fome, no ambiente domiciliar, denota o estado de Insegurança Alimentar (IA), uma vez que contrapõe a dimensão "acesso aos alimentos" do conceito de SAN. A Escala Brasileira de Insegurança Alimentar (EBIA) é um instrumento psicométrico que tem alta validade para o diagnóstico da IA no Brasil [MDS-SAGI 2014], e sua aplicação prática ocorre na Pesquisa Nacional por Amostra de Domicílios (PNAD) desde 2004. Em 2013, os resultados divulgados pela PNAD enfatizam alta prevalência de IA em populações que apresentam vulnerabilidades, principalmente, relativas às questões alimentares e aos determinantes socioeconômicos [Trivellato et al. 2019].

No atual contexto de saúde pública, cujo cenário é de pandemia, para além das questões relativas ao adoecimento da população pela contaminação viral, outras mazelas evidenciam-se. Um trecho do Comunicado 01, publicado pelo Fórum Brasileiro de Soberania e Segurança Alimentar e Nutricional (FBSSAN), merece destaque [FBSSAN 2020, pg. 01]: "A Pandemia escancara as desigualdades sociais, étnico-raciais e de gênero e as condições precárias de vida a que estão submetidas parcelas imensas da população brasileira. Evidencia a grave crise politico-institucional, o desmonte dos sistemas de saúde e proteção social e a paralisação de praticamente todos os programas de segurança alimentar e nutricional (SAN), o que compromete a capacidade e agilidade das respostas emergenciais diante da crise”. As reflexões oriundas do referido Comunicado fazem ecoar uma mensagem vinculada à urgência de ações que possam garantir o direito à alimentação e combater a fome, partindo da premissa que a vida e a dignidade humana devem estar em primeiro lugar.

O projeto apresentado neste artigo tem como contexto de atuação o município de Lavras/MG, especificamente, as ações realizadas pelo Banco Municipal de Alimentos (BMAL). O BMAL foi criado em abril/2019 pela Secretaria Municipal de Desenvolvimento Social, em parceria com o Departamento de Nutrição da Universidade de Lavras e do Coletivo de Segurança Alimentar e Nutricional Sustentável de Lavras/MG (SANS Lavras/MG). O BMAL funciona como uma central de recepção das doações de alimentos do município e realiza o trabalho de seleção e de encaminhamento dos gêneros para as entidades socioassistenciais cadastradas e para as famílias em situação de vulnerabilidade social, referenciadas pelos Centros de Referências em Assistência Social (CRAS). Atualmente, o BMAL possui 16 entidades cadastradas e atende os 6 (seis) CRAS do município.

A avaliação da situação familiar é realizada pelos técnicos dos CRAS (psicólogos e assistentes sociais), a partir de critérios socioeconômicos, tais como, renda, condição de moradia e membros familiares classificados dentro de grupos de

\footnotetext{
${ }^{1}$ Compreende-se sistema alimentar como o processo que abrange desde o acesso à terra, à água e aos meios de produção, às formas de processamento, de abastecimento, de comercialização e de distribuição à escolha e consumo dos alimentos, incluindo as práticas alimentares individuais e coletivas, até a geração e a destinação de resíduos [MDS-SAGI 2012].
} 
risco (idosos, crianças, portadores de doenças crônicas). Embora seja o instrumento oficial utilizado para avaliar a insegurança alimentar no país, a EBIA não é utilizada de forma padronizada dentro do protocolo de avaliação familiar feita pelos técnicos dos CRAS do município. A utilização da EBIA possibilita a classificação familiar segundo o nível de insegurança alimentar, servindo de medida para qualificar o tipo de assistência que precisa ser fornecida para a família, e como instrumento para acompanhar a evolução da situação de insegurança alimentar.

Neste trabalho, o objetivo é conhecer a dinâmica das famílias assistidas pelos CRAS, a partir de um recurso mobile, com foco na percepção e na vivência de insegurança alimentar e fome no nível domiciliar. Para isso, foram estabelecidos os seguintes objetivos específicos: i) Implementar uma plataforma computacional denominada Di@ (Direito à Alimentação) na rotina de trabalho dos CRAS, de modo a facilitar a operacionalização do processo de identificação das famílias assistidas; ii) Identificar mecanismos de priorização das famílias que enfrentam algum nível de insegurança alimentar, permitindo que os alimentos (cestas básica e cestas verdes) cheguem às famílias de forma eficiente; iii) Implementar rotinas de acompanhamento longitudinal do nível de insegurança alimentar das famílias assistidas, a partir da reaplicação da EBIA a cada 3 meses; e iv) Impulsionar, a partir do uso da Plataforma Di@, a elaboração de instrumentos válidos para a avaliação de processo das ações do Banco Municipal de Alimentos.

Este artigo está organizado da seguinte forma. Na Seção 2, os requisitos e a arquitetura da Plataforma Di@ são apresentados. Na Seção 3, a Plataforma Di@ é descrita com mais detalhes, ressaltando suas principais funções. $\mathrm{Na}$ Seção 4, os resultados preliminares de uma avaliação realizada com a Plataforma Di@ são descritos. Na Seção 5, são apresentadas as considerações finais.

\section{Levantamento dos Requisitos e Arquitetura da Plataforma Di@}

A Plataforma Di@ é um recurso inovador e inédito que foi desenvolvida em decorrência de uma demanda premente do município de Lavras/MG, a partir da seguinte questão: "Como estabelecer requisitos de priorização entre famílias assistidas pelos CRAS para destinar e controlar os beneficios Cestas Básica e Cestas Verdes?”.

A partir dessa questão, foram mobilizados o Departamento de Nutrição, o Departamento de Ciência da Computação da Universidade Federal de Lavras (UFLA), o Banco de Alimentos da Secretaria Municipal de Desenvolvimento Social e um CRAS do município para construção da linha de base e produtos do projeto.

O desenvolvimento da Plataforma Di@ iniciou-se com levantamento de requisitos, por meio de entrevistas realizadas com uma funcionária do CRAS, uma professora e uma aluna de pós-graduação do Departamento de Nutrição da UFLA. As entrevistas foram realizadas, virtualmente, por meio do aplicativo de troca de mensagens Whatsapp. A partir desse levantamento, chegou-se aos seguintes requisitos funcionais, isto é, requisitos relacionados ao que o sistema deve fazer e como ele deve se comportar diante de alguns cenários:

1. A Plataforma DI@ deve permitir o cadastramento de técnicos dos CRAS. As informações a serem cadastradas são nome, e-mail e senha; 
2. A Plataforma Di@ deve permitir a manutenção (inclusão, recuperação, atualização e remoção) de entrevistas com famílias em situação de insegurança alimentar, por parte dos técnicos dos CRAS. Cada entrevista deve conter as seguintes informações (as informações com “*” são de caráter obrigatório para o cadastro): nome* e CPF* do chefe de família, Endereço*, Telefone Fixo, Telefone Celular, Número de Identificação Social (NIS), Renda Familiar*, Quantidade de Membros Familiares Total*, Quantidade de Membros Familiares de 0 a 2 anos, Quantidade de Membros Familiares de 2 a 5 anos, Quantidade de Membros Familiares de 5 a 18 anos, Quantidade de Gestantes, Quantidade Deficientes, Quantidade de Idosos, Programas e Projetos de Assistência pelos quais a Família foi Contemplada (BPC, bolsa família, pensão por morte, pensão alimentícia, entre outros) e Respostas para as perguntas da EBIA (Tabela 1);

3. A Plataforma Di@ deve permitir a exportação das informações contidas nas entrevistas em formato compatível com sistemas de software de planilha eletrônica e em arquivo PDF, para posterior arquivamento no CRAS.

Tabela 1. Escala Brasileira de Insegurança Alimentar

\begin{tabular}{l}
\hline \multicolumn{1}{|c|}{ Perguntas Gerais } \\
\hline $\begin{array}{l}\text { Os moradores do seu domicílio tiveram a preocupação de que a comida acabasse antes que } \\
\text { tivessem dinheiro para comprar mais comida? }\end{array}$ \\
\hline $\begin{array}{l}\text { Os alimentos acabaram antes que os moradores desse domicílio tivessem dinheiro para } \\
\text { comprar mais comida? }\end{array}$ \\
\hline $\begin{array}{l}\text { Os moradores desse domicílio ficaram sem dinheiro para ter uma alimentação saudável e } \\
\text { variada? }\end{array}$ \\
\hline $\begin{array}{l}\text { Os moradores deste domicílio comeram apenas alguns poucos tipos de alimentos que ainda } \\
\text { tinham, porque o dinheiro acabou? }\end{array}$ \\
\hline $\begin{array}{l}\text { Algum adulto/idoso deixou de fazer alguma refeição porque não havia dinheiro para comprar } \\
\text { a comida? }\end{array}$ \\
\hline $\begin{array}{l}\text { Algum adulto/idoso comeu menos do que achou que devia, porque não havia dinheiro para } \\
\text { comprar comida? }\end{array}$ \\
\hline $\begin{array}{l}\text { Algum adulto/idoso sentiu fome, mas não comeu, porque não tinha dinheiro para comprar } \\
\text { comida? }\end{array}$ \\
\hline $\begin{array}{l}\text { Algum adulto/idoso ficou um dia inteiro sem comer ou, teve apenas uma refeição ao dia, } \\
\text { porque não tinha dinheiro para comprar a comida? }\end{array}$ \\
\hline \multicolumn{1}{|c|}{ Perguntas Específicas para MENORES de 18 Anos } \\
\hline $\begin{array}{l}\text { As crianças/adolescentes não puderam ter uma alimentação saudável e variada, porque não } \\
\text { havia dinheiro para comprar comida? }\end{array}$ \\
\hline $\begin{array}{l}\text { As crianças/adolescentes comeram apenas alguns poucos tipos de alimentos que ainda havia } \\
\text { neste domicílio, porque o dinheiro acabou? }\end{array}$ \\
\hline $\begin{array}{l}\text { Alguma criança/adolescente comeu menos do que você achou que devia, porque não havia } \\
\text { dinheiro para comprar a comida? }\end{array}$ \\
\hline $\begin{array}{l}\text { Foi diminuída a quantidade de alimentos das refeições de alguma criança/adolescente, porque } \\
\text { não havia dinheiro suficiente para comprar a comida? }\end{array}$ \\
\hline $\begin{array}{l}\text { Alguma criança/adolescente deixou de fazer alguma refeição, porque não havia dinheiro para } \\
\text { comprar a comida? }\end{array}$ \\
\hline $\begin{array}{l}\text { Alguma criança/adolescente sentiu fome, mas não comeu porque não havia dinheiro para } \\
\text { comprar mais comida? }\end{array}$ \\
\hline
\end{tabular}


Além dos requisitos anteriores, há alguns requisitos não-funcionais, isto é, restrições existentes sobre a funcionalidade da Plataforma Di@:

1. A Plataforma Di@ deve permitir a manutenção (inclusão, recuperação, atualização e remoção) de entrevistas, sem necessidade de acesso à Internet e sem a necessidade de autenticação, por parte dos técnicos dos CRAS;

2. A Plataforma Di@ deve permitir o envio de entrevistas cadastradas a uma base de dados central, quando houver acesso à Internet e mediante informações de login e senha por parte dos técnicos dos CRAS;

3. A Plataforma Di@ deve impedir que entrevistas enviadas à base de dados sejam editadas pelos técnicos dos CRAS, visando garantir a consistência dos dados das entrevistas;

4. A Plataforma Di@ deve permitir que entrevistas enviadas à base de dados sejam desbloqueadas para edição e posterior envio ao servidor. A entrevista anterior não deve ser sobrescrita no servidor, mas uma nova entrevista deverá ser incluída. Isso é necessário, pois uma família pode ser revisitada futuramente e o técnico do CRAS poderá apenas atualizar seus dados, sem precisar preencher todo formulário da entrevista novamente.

Diante dos requisitos elencados anteriormente, optou-se pelo desenvolvimento de duas aplicações distintas para compor a Plataforma Di@: i) Sistema Di@ Servidor, responsável pela manutenção da base de dados de entrevistas e de usuários do sistema (requisitos 1 e 3); e ii) Sistema Di@ - Cliente, responsável pela manutenção das entrevistas, por parte dos técnicos dos CRAS (requisitos 2, 4, 5, 6 e 7). Na Figura 1, é apresentado um esquema geral da arquitetura da Plataforma Di@. Os técnicos dos CRAS podem usar o Sistema Di@ - Cliente em seus dispositivos móveis (por exemplo, celulares e tablets) para registrar, manter e enviar as entrevistas ao Sistema Di@ Servidor. No Sistema Di@ - Servidor, o gerente/administrador do CRAS consegue obter relatórios gerenciais, a partir dos dados enviados pelo Sistema Di@ - Cliente.

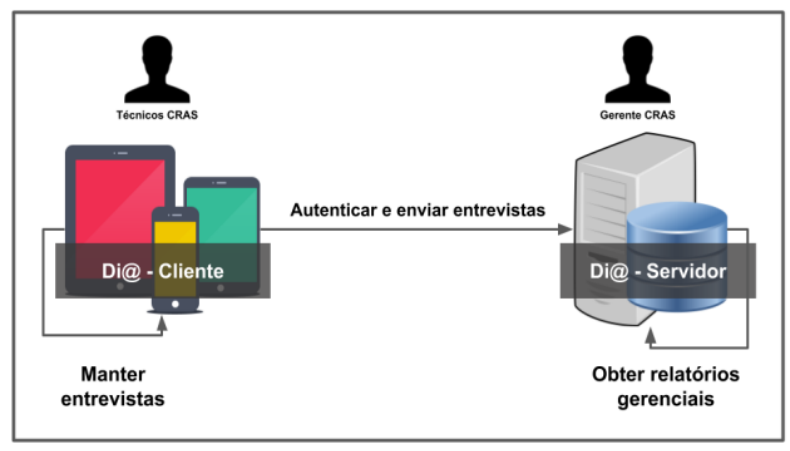

Figura 1. Arquitetura da Plataforma Di@

Para o desenvolvimento do Sistema Di@ - Cliente, a tecnologia PWA (Progressive Web App) foi utilizada. Essa tecnologia combina recursos oferecidos pelos mais modernos navegadores com as vantagens de uso de um celular. Dentre as principais características desse tipo de tecnologia, as que mais se alinham às demandas deste projeto são: i) responsividade, ou seja, um aplicativo PWA se adequa a diversos tipos de formato de tela, tais como, desktop, celular e tablet; e ii) independência de conectividade, uma vez que um aplicativo PWA pode trabalhar totalmente off-line ou em redes de baixa qualidade. Para implementação do PWA, foi utilizado o framework 
Ionic $^{2}$, uma das principais ferramentas para desenvolvimento de aplicativos híbridos disponíveis no mercado.

Quanto ao Sistema Di@ - Servidor, foram utilizados a linguagem de programação PHP (PHP: Hypertext Preprocessor), o framework Laravel $^{3}$ e o sistema gerenciador de banco de dados $\mathrm{MySQL}^{4}$. Tais tecnologias foram escolhidas, pois: i) possuem boa documentação e comunidade de usuários; ii) permitem o desenvolvimento de aplicações livres (open-source); e iii) são conhecidas pelos desenvolvedores envolvidos neste projeto.

É importante mencionar que o Sistema Di@ - Cliente e o Sistema Di@ Servidor são sistemas de software livre e estão disponíveis em repositório público ${ }^{5}$. Para o desenvolvimento da Plataforma Di@, foi utilizado um modelo iterativo e incremental de desenvolvimento de sistemas de software, no qual pequenas porções do sistema eram implementadas, entregues e validadas pelo grupo de colaboradoras previamente listadas.

\section{A Plataforma Di@}

Todos os requisitos elencados na Seção 3 foram implementados e encontram-se em fase de teste final. Nesta seção, são apresentadas algumas telas da Plataforma Di@ para demonstrar a sua funcionalidade.

O cenário comum de uso da Plataforma Di@ inicia com a inserção (cadastramento) de um técnico do CRAS no Sistema Di@ - Servidor. Isso é importante, pois o técnico precisará de credenciais de acesso ao Sistema Di@ para enviar as entrevistas realizadas por ele. Após o cadastramento, o técnico pode acessar o Sistema Di@ - Cliente em seu computador ou dispositivo móvel.

Na Figura 2, é apresentada a tela inicial do Sistema Di@ - Cliente. Nela, o técnico pode visualizar as entrevistas cadastradas por ele. Para cada entrevista, são exibidos o nome do chefe de família, o endereço da família, a data em que foi realizada a entrevista e um ícone, indicando se a entrevista foi enviada ao Sistema Di@ -

Servidor ( $W$ ) ou não $(\checkmark)$ ). Para criar uma entrevista, o técnico do CRAS deve clicar sobre o botão "Nova", que aparece no canto superior direito da tela da Figura 2.

Na Figura 3, por sua vez, é apresentada a tela de inclusão (cadastro) de uma entrevista. Ela contém os campos necessários para uma entrevista, conforme descrito no requisito 2 (Seção 3). Algumas características importantes dessa tela são: i) o sistema realiza a validação de campos obrigatórios; ii) o botão "Salvar", no canto superior direito da tela, fica habilitado apenas quando os requisitos obrigatórios para inclusão (cadastramento) de uma entrevista são atendidos; e iii) há botões de atalho na parte superior da tela, que oferecem ao técnico do CRAS a possibilidade de "navegar" entre seções específicas do formulário de cadastro de uma entrevista.

Na Figura 4, são apresentadas as ações que podem ser realizadas sobre uma entrevista cadastrada no Sistema Di@ - Cliente: i) Visualizar: apresenta as

\footnotetext{
${ }^{2}$ https://ionicframework.com/

${ }^{3}$ https://laravel.com/

${ }^{4}$ https://www.mysql.com/

${ }^{5} \mathrm{https}: / /$ github.com/direito-alimentacao/
} 
informações incluídas (cadastradas) para a entrevista em questão; ii) Editar: permite ao técnico corrigir ou completar qualquer informação a respeito de uma entrevista, desde que ela não tenha sido enviada ao Sistema Di@ - Servidor (requisito 6 - Seção 3); iii) Enviar: transmite os dados da entrevista realizada para o Sistema Di@-Servidor; iv) Desbloquear: faz com que a entrevista fique com status de não-enviada ao servidor para o técnico do CRAS poder editá-la e enviá-la novamente ao servidor. Contudo, a entrevista enviada anteriormente não será sobrescrita, mas uma nova entrevista será incluída (cadastrada); e v) Remover: apaga o registro da entrevista do dispositivo do usuário (essa ação não afeta os dados enviados ao Sistema Di@ - Servidor).

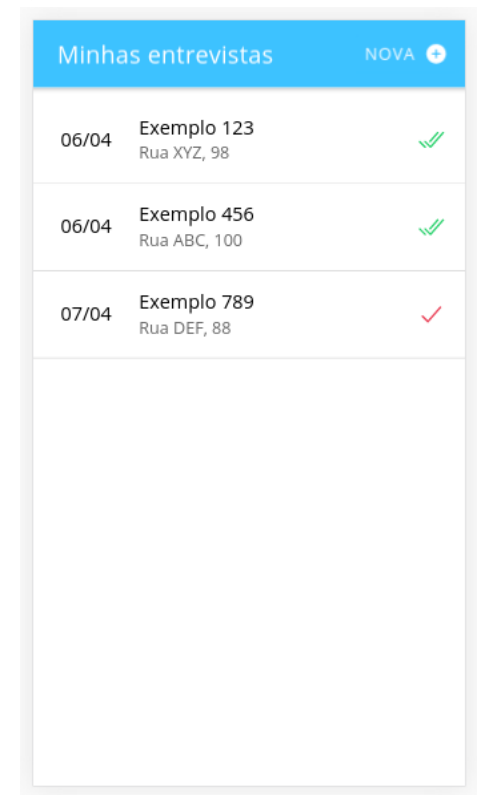

Figura 2. Tela inicial do Sistema Di@ - Cliente

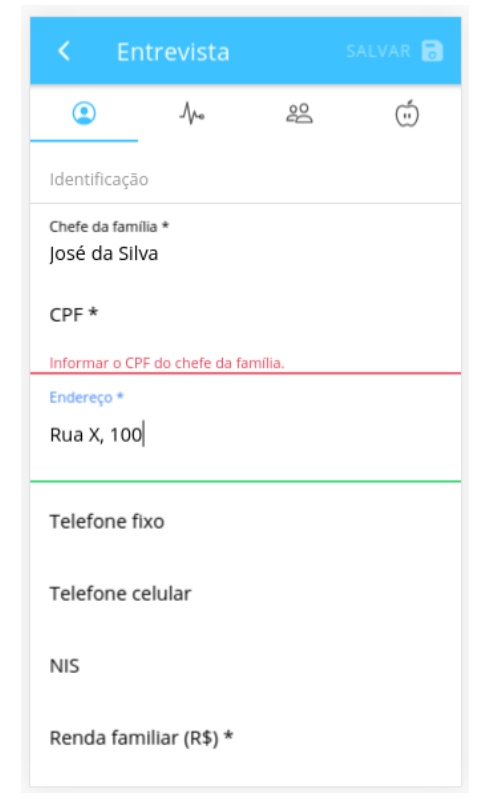

Figura 3. Tela de inclusão de uma entrevista no Sistema Di@ Cliente

Uma vez que as entrevistas foram enviadas ao Sistema Di@ - Servidor, o administrador do CRAS pode ter acesso a essas informações. Na Figura 5, é apresentada a tela inicial do Sistema Di@ - Servidor, na qual são apresentadas as entrevistas enviadas pelos técnicos.

A partir dessa tela, o administrador do CRAS pode visualizar os dados de cada entrevista, individualmente (botão Visualizar), remover uma entrevista (botão Excluir), gerar um arquivo PDF com os dados da tabela apresentada na Figura 5 (botão Gerar PDF), exportar os dados das entrevistas para um arquivo compatível com sistemas de software de planilha eletrônica (botão Exportar para Excel) e filtrar o conteúdo da tabela apresentada por data em que as entrevistas foram realizadas (botão Filtrar).

Na Figura 6, é apresentada uma parte da tela de visualização das informações de uma entrevista específica. Nessa tela, é possível gerar um arquivo PDF contendo os dados da entrevista realizada para posterior impressão e arquivamento no CRAS (botão Imprimir Cadastro). Um vídeo explicativo, com a utilização da funcionalidade do Sistema Di@, pode ser visto em https://www.youtube.com/watch?v=wRiZEUqVUnc. 


\section{Resultados Preliminares}

Para avaliação da Plataforma Di@, uma avaliação foi realizada por meio de questionários elaborados com base no modelo de aceitação de tecnologia (TAM Technology Acceptance Model). Esse modelo visa prover indícios sobre a utilidade e a facilidade de uso percebida pelos usuários da aplicação [Davis 1993] e possui como objetivo explicar o comportamento das pessoas em relação à aceitação de uma tecnologia. Para isso, sugere-se a criação de um questionário, ao qual são atribuídas afirmações relacionadas à utilidade e à facilidade de uso percebidas pelos usuários da tecnologia em análise. Para cada afirmação, o respondente deve manifestar sua concordância por meio de respostas gradativas com escala do tipo Likert.

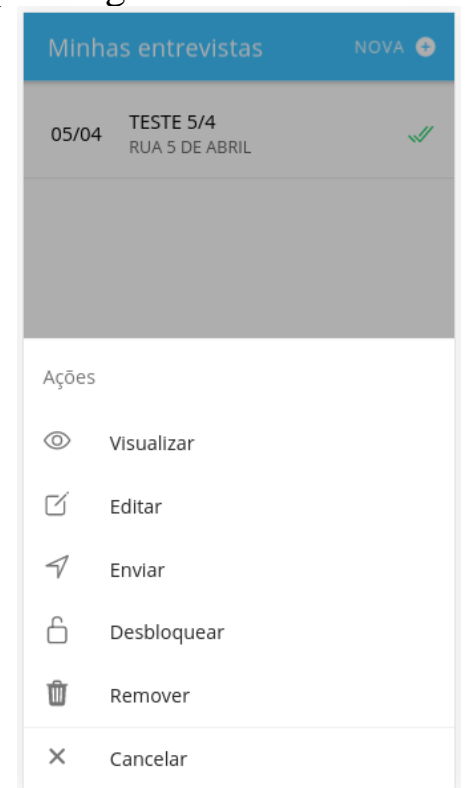

Figura 4. Ações que podem ser realizadas sobre uma entrevista no Sistema Di@ - Cliente

Direito a Alimentação

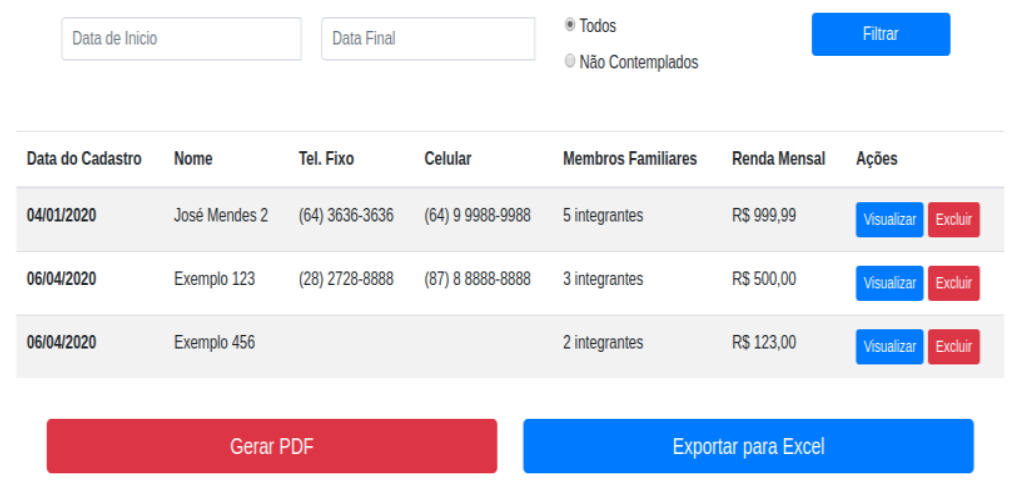

Figura 5. Tela inicial do Sistema Di@ - Servidor 
Nesta seção, os resultados preliminares ${ }^{6}$ de uma avaliação realizada com a Plataforma Di@, por meio do modelo TAM, são apresentados. De acordo com esta avaliação inicial, $67 \%$ dos usuários descreveram a atividade de manutenção de entrevistas como muito fácil e 33\% como fácil. O mesmo cenário ocorre para as funções de autenticação de usuário e de envio de uma entrevista ao CRAS. Quanto ao gerenciamento de entrevistas no Sistema Di@ - Servidor, 66\% dos usuários descrevem essa atividade como muito fácil, 17\%, como fácil e 17\% como nem fácil, nem difícil.

Um aspecto que chamou a atenção foi que $17 \%$ dos usuários consideraram que a Plataforma Di@ tornaria o processo de realização de entrevistas com as famílias difícil e deveria ser feito por um profissional da nutrição, a saber:

"Primeiramente gostaria de entender o objetivo da entrevista, uma vez que a situação emergencial que estavamos (sic) vivendo a aplicação do questionário dificulta o trabalho do profissional e constrange o usuário";

“A perguntas são muito repetitivas. E penso que deve ser realizado por um profissional Voltar a Página Inicial da nutrição".

Identificação

\begin{tabular}{|l|l|}
\hline Nome do Chefe da Familia & Data do Cadastro \\
\hline Exemplo 123 & $06 / 04 / 2020$ \\
\hline Endereço Completo & \\
\hline Rua XYZ, 98 & NIS \\
\hline CPF & 12323233333 \\
\hline $878.888 .888-78$ & Telefone Celular \\
\hline Telefone Fixo & $(87) 888888-8888$ \\
\hline (28) 2728-8888 & Renda Familiar \\
\hline Membros Familiares & R $\$ 500$ \\
\hline 3 Integrantes & \\
\hline
\end{tabular}

Figura 6. Tela de visualização de uma entrevista do Sistema Di@ - Servidor

A partir disso, entende-se que, como trabalho futuro, é preciso haver um projeto de conscientização e de integração dos técnicos dos CRAS no processo de desenvolvimento da Plataforma Di@, visando melhor entendimento dos objetivos do projeto e, consequentemente, do engajamento desse público-alvo à Plataforma Di@. Uma iniciativa realizada na direção desse objetivo foi desenvolver um vídeo de apresentação dos objetivos do projeto a ser divulgado aos CRAS interessados em usar a PlataformaDi@. Um comentário positivo, deixado por um usuário avaliador foi:

"Achei o aplicativo fácil de usar, adaptável às possíveis dificuldades do dia a dia, como a falta de internet em alguns territórios. A possibilidade de exportar em Excel facilita $o$ processo para avaliação das informações e produção de relatórios".

\footnotetext{
6 Apenas os resultados mais relevantes são apresentados nesta seção. A versão completa com os resultados pode ser acessada na planilha: https://docs.google.com/ spreadsheets/d/1VoqA7mFicQ6aOHP0kVekMtLy_mZL9Jd55Mm-X9DiMA4/edit?usp=sharing
} 
Isso mostra que a Plataforma Di@ vai ao encontro de uma demanda real. Além disso, a Plataforma Di@ pode trazer benefícios para os profissionais atuantes na área de (in)segurança alimentar, ao permitir a extração de dados estatísticos sobre os atendimentos realizados às famílias.

\section{Considerações Finais}

O conjunto de vivências experimentadas, até o momento, por este projeto, indica a potencialidade de atividades transdisciplinares, intersetoriais e multiprofissionais, com convergência temática nas demandas sociais. Outros resultados ainda estão por vir, contudo são conhecidas algumas necessidades e/ou recomendações para o projeto e seus produtos terem utilidade a longo prazo, quais sejam: i) sendo comprovado o impacto positivo da Plataforma Di@ nas rotinas de trabalho do Banco Municipal de Alimentos e CRAS, sugere-se a sua replicação para outros municípios e realidades sociais e culturais, inclusive com a exploração de outras informações e instrumentos de avaliação; ii) recomenda-se a realização de um estudo adicional sobre a usabilidade do Sistema Di@ - Cliente e do Sistema Di@ - Servidor, considerando o grau de facilidade/dificuldade que o usuário tem para entender o que está sendo apresentado, grau de satisfação e nível de conforto para alcance dos objetivos; e iii) sugere-se que a Plataforma Di@ possa ser incorporada, como recurso digital, na rotina de trabalho e avaliação da Secretaria Municipal do Desenvolvimento Social.

\section{Referências}

Brasil. "Lei $\mathrm{n}^{\mathrm{o}}$ 11.346, de 15 de Setembro de 2006". 2006. Disponível em: http://www.planalto.gov.br/ccivil_03/_Ato2004-2006/2006/Lei/L11346.htm. Acessado em: Abril/2020.

Davis F.D. "User acceptance of information technology: systemcharacteristics, user preceptions and behavioral impacts". 1993. Int J Man MachStud 38:475-48.

FBSSAN. "Adiada a Conferência Popular por Direitos, Democracia, Soberania e SAN: seguimos mobilizados no enfrentamento do Coronavírus". 2020. Disponível em: https://fbssan.org.br/2020/02/adiamentodaconferencia/. Acessado em: Abril/2020.

MDS/SAGI. Ministério do Desenvolvimento Social e Combate à Fome e Secretaria de Avaliação e Gestão da Informação. "Marco de Referência de Educação Alimentar e Nutricional para as Políticas Públicas". 2012. Disponível em: http://www.mds.gov.br/webarquivos/arquivo/seguranca_alimentar/caisan/Publicacao/Educac ao_Alimentar_Nutricional/1_marcoEAN.pdf. Acessado em: Abril/2020.

MDS/SAGI. Ministério do Desenvolvimento Social e Combate à Fome e Secretaria de Avaliação e Gestão da Informação. "Escala Brasileira de Insegurança Alimentar - EBIA: análise psicométrica de uma dimensão da Segurança Alimentar e Nutricional". 2014. Disponível em: https://fpabramo.org.br/acervosocial/wpcontent/uploads/sites/7/2017/08/328.pdf. Acessado em: Abril/2020.

Panelli-Martins, B. E. P.; dos Santos, S. M. C.; Assis A. M. O. "Segurança alimentar e nutricional: desenvolvimento de indicadores e experimentação em um município da Bahia, Brasil". Rev. Nutr., Campinas, 21(Suplemento):65s-81s, jul./ago., 2008.

Trivellato, P. T.; Morais, D. C.; Lopes, S. O.; Miguel, E. S.; Franceschini S. C. C.; Priore, S. E. "Insegurança alimentar e nutricional em famílias do meio rural brasileiro: revisão sistemática". Ciência \& Saúde Coletiva, 24(3):865-874, 2019. 